\title{
Control Design for Attitude and Position Based on Real Time Color Tracking System with Image Processing Technique
}

\author{
Hsu Myat Tin Swe ${ }^{1}$, Hla Myo Tun ${ }^{2} \&$ Maung Maung Latt ${ }^{3}$ \\ ${ }^{1,2}$ Department of Electronic Engineering, Yangon Technological University, Yangon, Myanmar \\ ${ }^{1,3}$ Department of Electronic Engineering, Technological University (Taungoo), Taungoo, Myanmar \\ Correspondence: Hla Myo Tun, Department of Electronic Engineering, Yangon Technological University, \\ Gyogone, Insein PO, 11011, Yangon, Myanmar.
}

Received: April 13, 2020

doi:10.5539/cis.v13n2p32
Accepted: April 22, 2020

Online Published: April 23, 2020

URL: https://doi.org/10.5539/cis.v13n2p32

\begin{abstract}
The paper mainly emphasizes on the control design for attitude and position based on real time color tracking system with image processing technique. The research problem in this study is to observe the high accuracy of the tracking system in image processing areas. The solution for this problem is to control the attitude and position of the object based on real time color tracking system. The objective of this study is to implement the image processing algorithms for autonomous tracking system. The specific objective of this study was fulfilled the experimental studies for contribution of real time color tracking for motion detection system in reality based on this study. This system is used the high performance camera to improve the enactment of tracking of a target and estimation of a motion. An image processing system consists of a light source to illuminate the sense, a sensor system, an interface between the sensor system and the computer. Then, color component analysis is used for color tracking system. MATLAB is competently used for tracking the ball and controlling the attitude and position of the ball.
\end{abstract}

Keywords: attitude and position control system, color tracking system, image processing, image detection, MATLAB simulation

\section{Introduction}

There are several presentations where it is beneficial to have an object that is proficient of position itself in a professional lacation relation to somewhat in the surroundings. Robotics is a very widespread field of knowledge. Since several years human beings have frustrated to construct autonomous robot. Robotics appeals ordinary people not only those educated in this territory. There are increasingly intricate robots fabricated. They are furnished with built-in computers consequently they developed autonomous organization. A diversity of sensors and locating approaches has been established over the year. Furthermost methods put your faith in energetic sensors which have assortment and power limitations, or count on reckoningally intricate approaches such as convalescing point from high-fidelity or optical flow. Detection is accomplished by using an exceptional implementation of Generalized Hough-Transform for which the forms are engendered by projecting the 3D-model into the image flat.

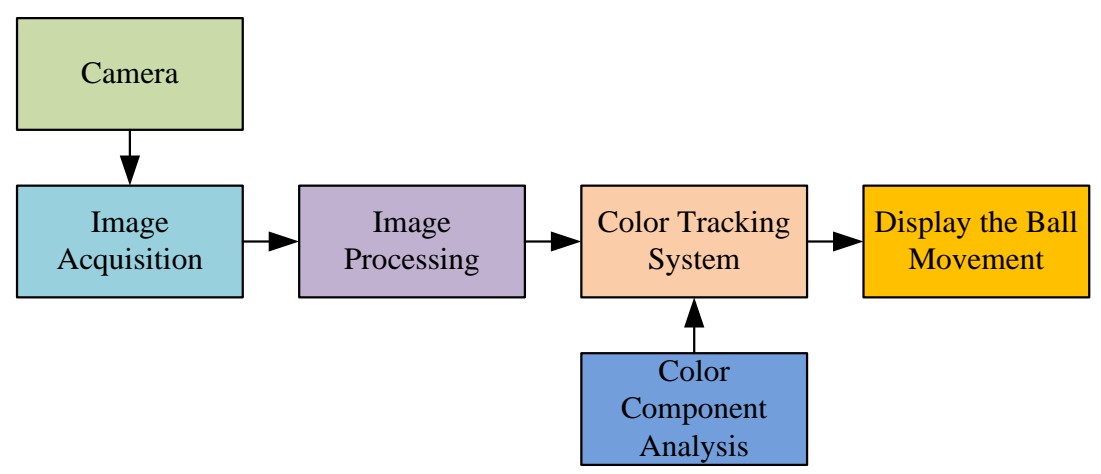

Figure 1. Block Diagram of Color Tracking System 
In the initialization part a reasonably extensive range of comparative positions and attitude between head and camera has to be verified for targeting for brilliance individuality, only information about the sign of the different between the predictable intensities on both sides of the edge of the polygons may be furthermore used in the GHT. Because the robots do not sense the real coordinate systems, it is obligatory to abate the location error with further matters in the environments. The tactic anticipated in this research work customs two orthogonal positioned cameras to identify color markers on the object. An object location and approach evaluator receipts the color spots location evidence on image flat as involvement and productivities the location and approach evidence in standardized coordinates practice. As a final point, the experimental results have revealed by using the MATLAB command. Figure.1 shows the process diagram of the color tracking system. In the image acquisition process, the movement of the orange ball is taken by the camera and avi file is saved in the computer hard disk. Then, the video file is processed by image processing techniques. The movement of ball is tracked by the color tracking system. After that the position of ball in each frame is tracked and robot starts to move following the ball. In this work, orange color ball is emphasized for the color analysis portion.

\section{Attitude and Position Control System}

In attitude and position control, dispersed controllers can be intended to consecutively stabilize the translational dynamics and the orientation dynamics of the moving object. An object location and approach evaluator earnings the color spots location evidence on image plane as involvement and productivities the location and approach evidence in standardized coordinate arrangement. Attitude and position control necessitates an approach and location sensor scheme of actuators to put on the apposite torque when compulsory and a set of reckoningnal set of rules and segments to expertise the actuators based on the sensor interpretations. The Control Systems for Attitude are essential in the maneuver of much contemporary period know-how. They are utilized on spaceship, airplane, and subsurface vehicles, and have a main protagonist in their appropriate maneuver. These schemes are also intended to effort independently with diminutive or no human mechanism, and have fool around a key title role in the conception and expansion of unmanned aerial systems, both flying and subsurface. Independent attitude control schemes work control arrangements that customarily necessitate mathematical reckonings to stream uninterrupted response to the vehicle's actuators. The archetypal and control arrangement being established in this study along with the test center research conducted will exploit enthusiastically obtainable expertise and be further achievable for an extensive variety of uses. The exhibiting and control arrangement will both be established using MATLAB environments, a matrix created work out program, which will formerly be used by a micro-computing maneuver. This research will afford the means for minor balance challenging and demonstation of approach control operations, provided that instructional and investigation prospects, and will oblige as a model for forthcoming improvements on minor scale approach control [Wie, B, Reston, and Virginia. 1998].

\section{Real-Time Color Tracking System}

There is a preprocessing stage before training the system. Image preprocessing is a common form for a variety of image operations. Image processing is necessary step in any image processing task involving the labeling and identification of constituent parts of an image or scene when it is needed to identify the number of items of a given color, size, or shape in an image. These algorithms generally are based on two foundation possessions of concentration standards:

- Discontinuity: to dividing wall an image based on unexpected variations in concentration (such as edge).

- Similarity: to dividing wall an image into constituencies comprising diverse sorts of texture, which are comparable as stated by a set of predefined benchmarks.

In the preprocessing step, input image is obtained via a digital camera or downloading from the internet. Signals are produced by the camera are stockpiled in the main image. The algorithms for image processing are accustomed excerpt a set of structures from the image to characterize an object. These images are resized by $150 \times 140$ pixels to get the same size of images. Then change the data type to the double floating type. To get the required outputs, the input images must have the same size of width and length in window [Tim Morris 2004, William K. Pratt 2000, Slawomir Bogumil Wesolkowski 1999, John C. Russ 1999, Miler. D.P. and Wright.A 1995].

\section{Image Segmentation Process}

Based on the general theory of thersholding, an input image from robot camera is thresholded to separate the ball from other surrounding objects and background. The original image is shown in Figure.2. This image is processed for thersholding by setting the value of $\mathrm{T}=1$ as shown in Figure.3. Since the threshold value is not 
enough to separate the ball from other objects, many white color objects are detected. When the value of threshold is increased, the ball is successfully extracted from surroundings and background as shown in Figure.4.

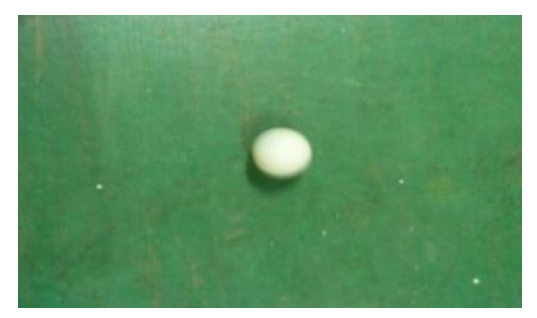

Figure 2. Original Image from Camera

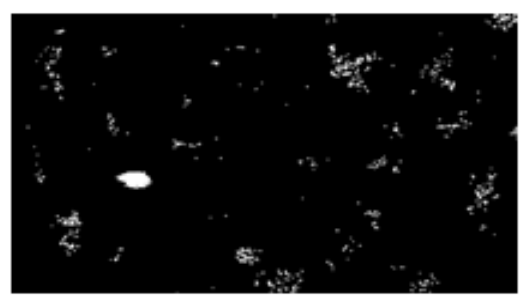

Figure 3. Output of Threshould with Insufficient Value of T

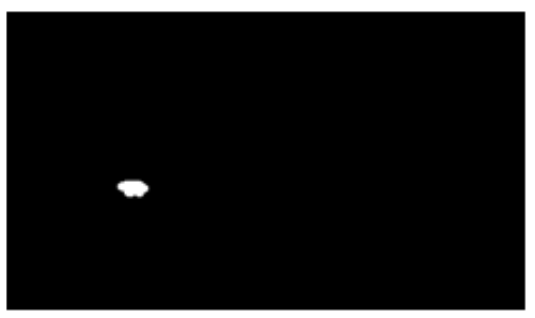

Figure 4. Output Image of Thresholding with Increased T

The results use optical flow estimation technique to estimate the motion vectors in each frame of the video sequence. By thresholding and performing morphological closing on the motion vectors, the binary feature images are produced. In this results, locate the ball in each binary feature image using blob analysis.

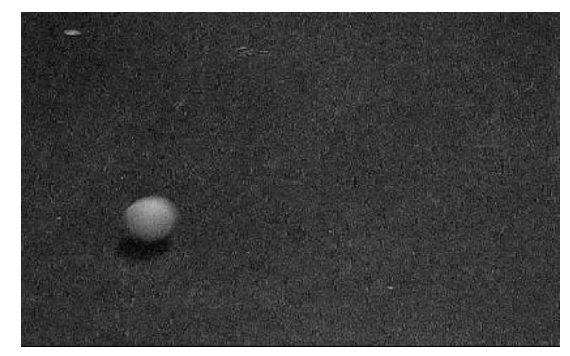

Figure 5. Original Image

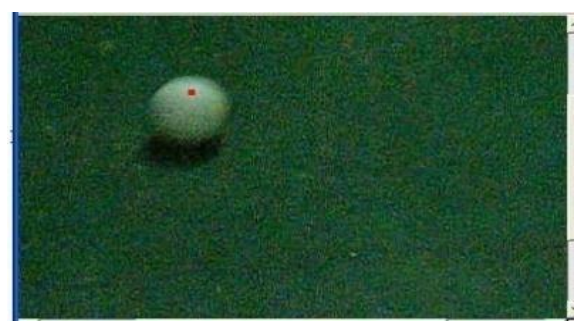

Figure 6. Video Frame after Some Processing Time 
Figure. 5 shows the original binary image captured by robot camera. Figure. 4 is the image after thresholding. Motion vectors are closed by morphological method in Fig.5. Target moving object is captured and tracked by a red dot in Figure.6. Image Segmentation necessitates the detachment or parting of the image into sections of comparable quality. The utmost uncomplicated quality for segmentation is image luminance generosity for a colorless image and color constituents for a color image. Image superiorities and texture are also convenient qualities for segmentation. The characterization of segmentation accepted in this study is intentionally preventive; no circumstantial evidence is consumed in the segmentation. Additionally, segmentation does not encompass categorizing every segment. The segmenter only segments an image; it does not endeavor to diagnose the separate segments or their associations to one additional. There is no concept of image segmentation. As significance, no single typical technique of image segmentation has appeared. Somewhat, there are an assortment of unplanned approaches that have established some degree of admiration. Since the approaches are unplanned, it would be valuable to obligate some means of evaluating their enactment. Haralick and Shapiro have recognized the succeeding qualitative recommendation for upright image segmentation: "Regions of image segmentation should be uniform and homogeneous with respect to some characteristic such as gray tone or texture. Region interiors should be simple and without many small holes. Adjacent regions of segmentation should have significantly different values with respect to the characteristic on which they are uniform. Boundaries of each segment should be simple, not ragged, and must be spatially accurate." Inappropriately, no measureable image segmentation enactment metric has been established.

\section{Implementation}

Figure. 7 shows the flowchart for color tracking system. Figure. 8 illustrates the flowchart of extract orange color. The furthermost conjoint image sensor used in cameras with electronic device is the charge coupled device. Another conjoint technique is by contour perusing. This comprises by means of a detector comprising one dimensional range of pixel.

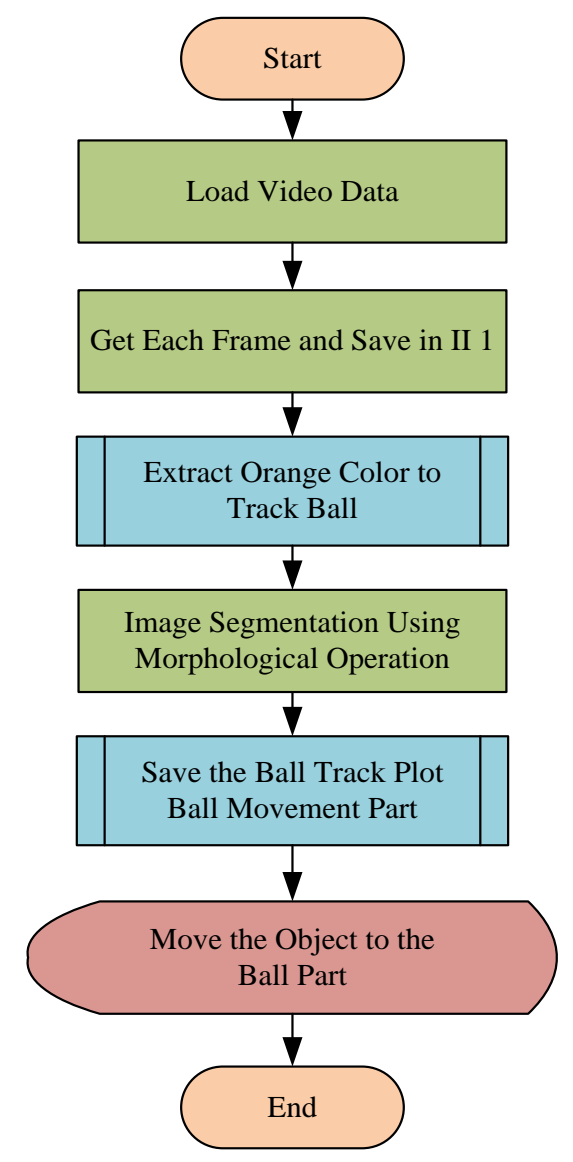

Figure 7. Flowchart for Color Tracking System 


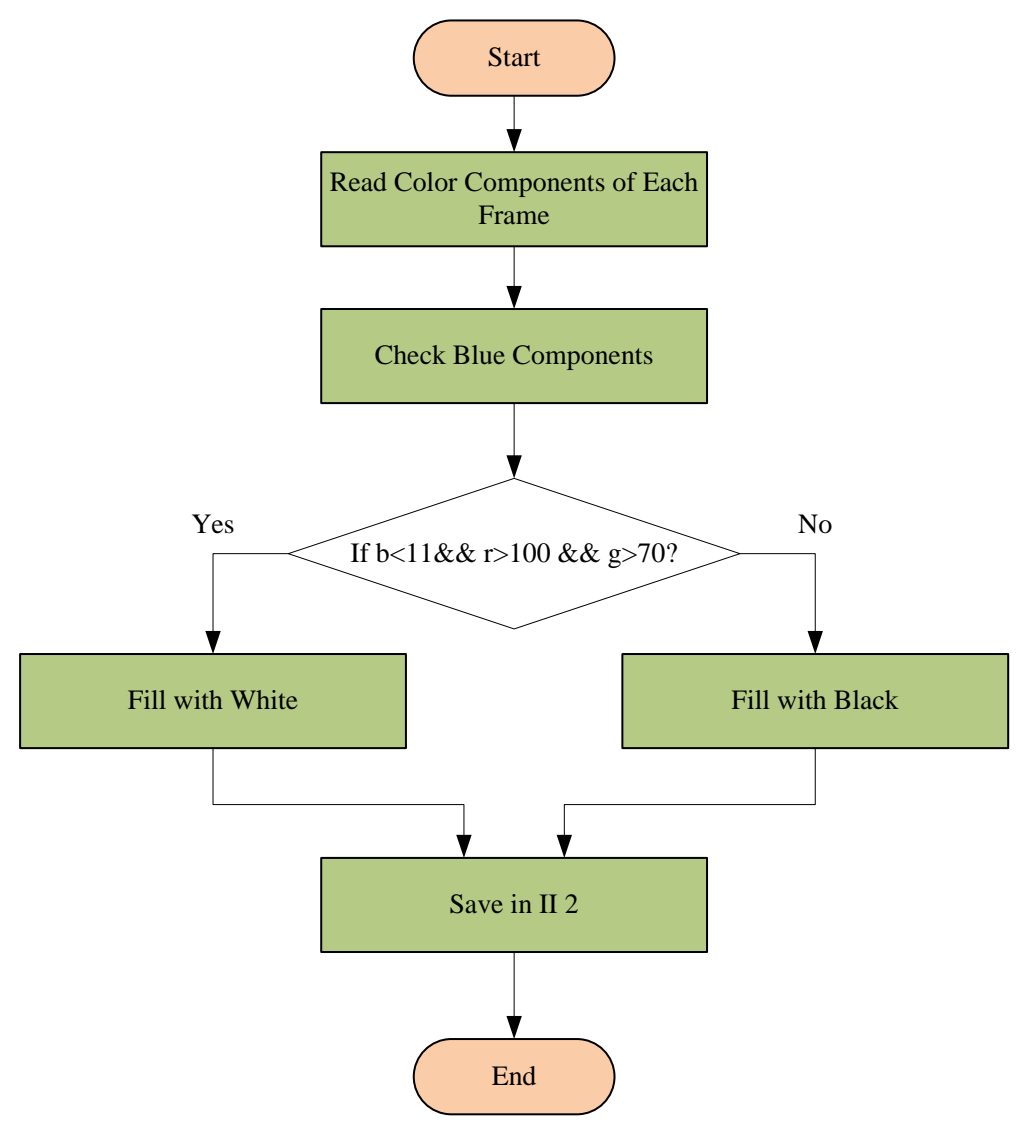

Figure 8. Flowchart of Extract Orange Color

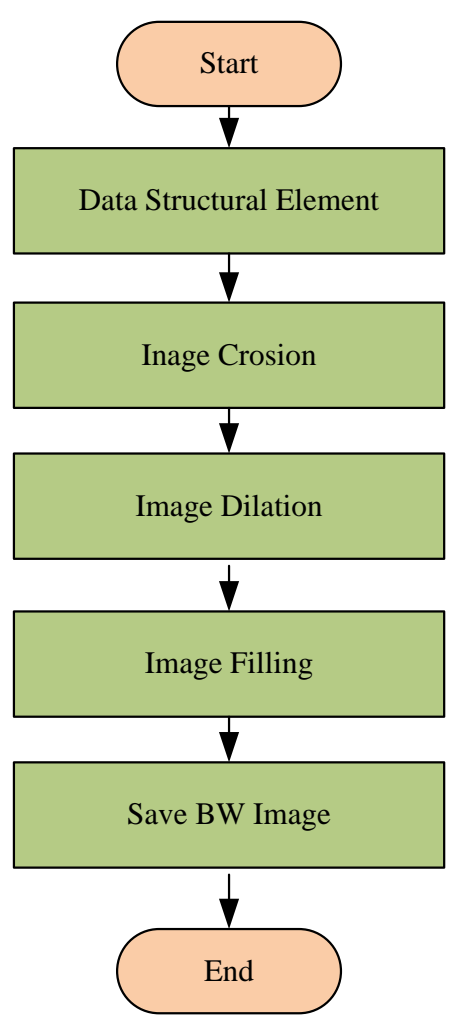

Figure 9. Flowchart of Image Segmentation 


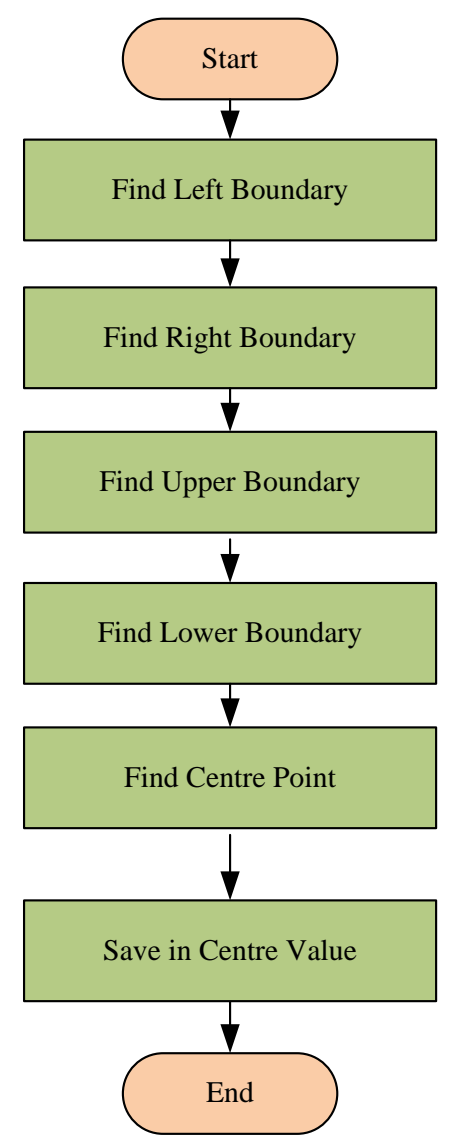

Figure 10. Flowchart of Finding Ball Movement Part

Figure.9 shows the flowchart of image segmentation. In image segmentation, structural element is firstly defined and then image erosion and dilation are performed. After that image filling is used to fill image regions and holes. Simulation in Figure10, to find the movement part of the ball, left boundary and right boundary are needed to find. And the upper and lower boundaries are also needed to find. After that center point is also finding.

\section{Simulation Results}

At the simulation program, two axes and 9 push buttons are constructed as shown in Figure.11. The push buttons are grouped in two parts: The first one is image processing purpose in color tracking of image and the second one is applied for display purpose. For display part, the user can select the desire type of image or tracked path at the push buttons.

In this work, the orange color is chosen as tracking color. So, if the orange color does not exist in the video file, the error message is displayed for the user selected file as shown in Figure.13. In each frame, Red, green and blue color components are detected for each pixel of image. If blue component $<11$ and red component is $>100$ and green component $>70$ - the color can be said that orange. If orange color is detected, the white is inserted for this pixel otherwise black color is inserted at non-orange color pixel.

If the show track pushbutton is clicked, the file open box is displayed as shown in Figure.14. And then, if the plot track pushbutton is clicked, the file open box is display as shown in Figure. 15.

If the show robot movement pushbutton is clicked, the file open box is displayed as shown in Figure.16. And Figure.17 showed the final position and direction of the ball. If the track path was from top to bottom, the direction angle showed the negative sign and if the track path was from bottom to top, the direction angle showed the positive sign.

After the ball path is saved in data file, it can be applied in Robot applications to track the path. The robot path can be illustrated in separate plot as shown in Figure.18. This track path is composed of square blocks in green color to be significant for user. 


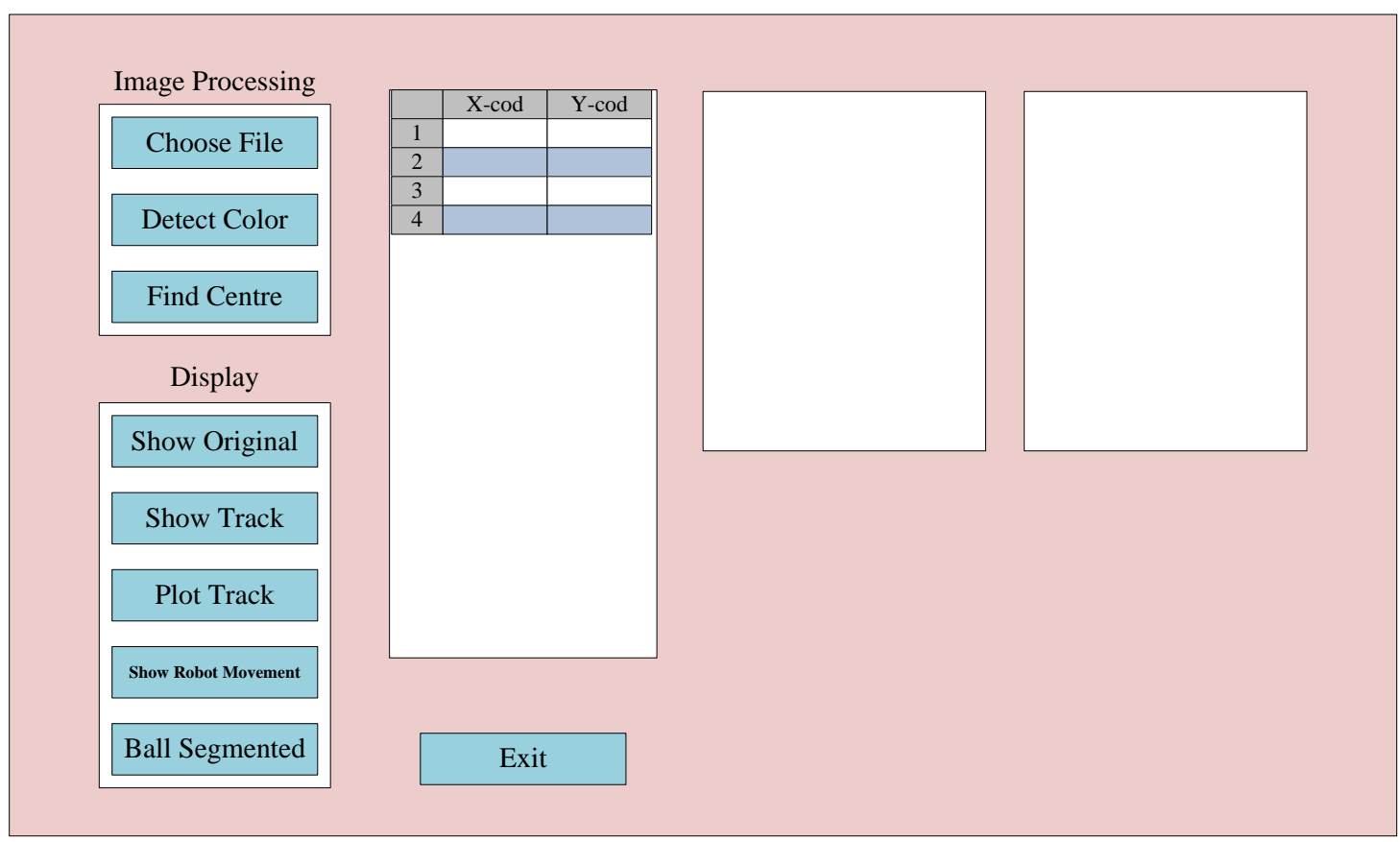

Figure 11. Menu Box

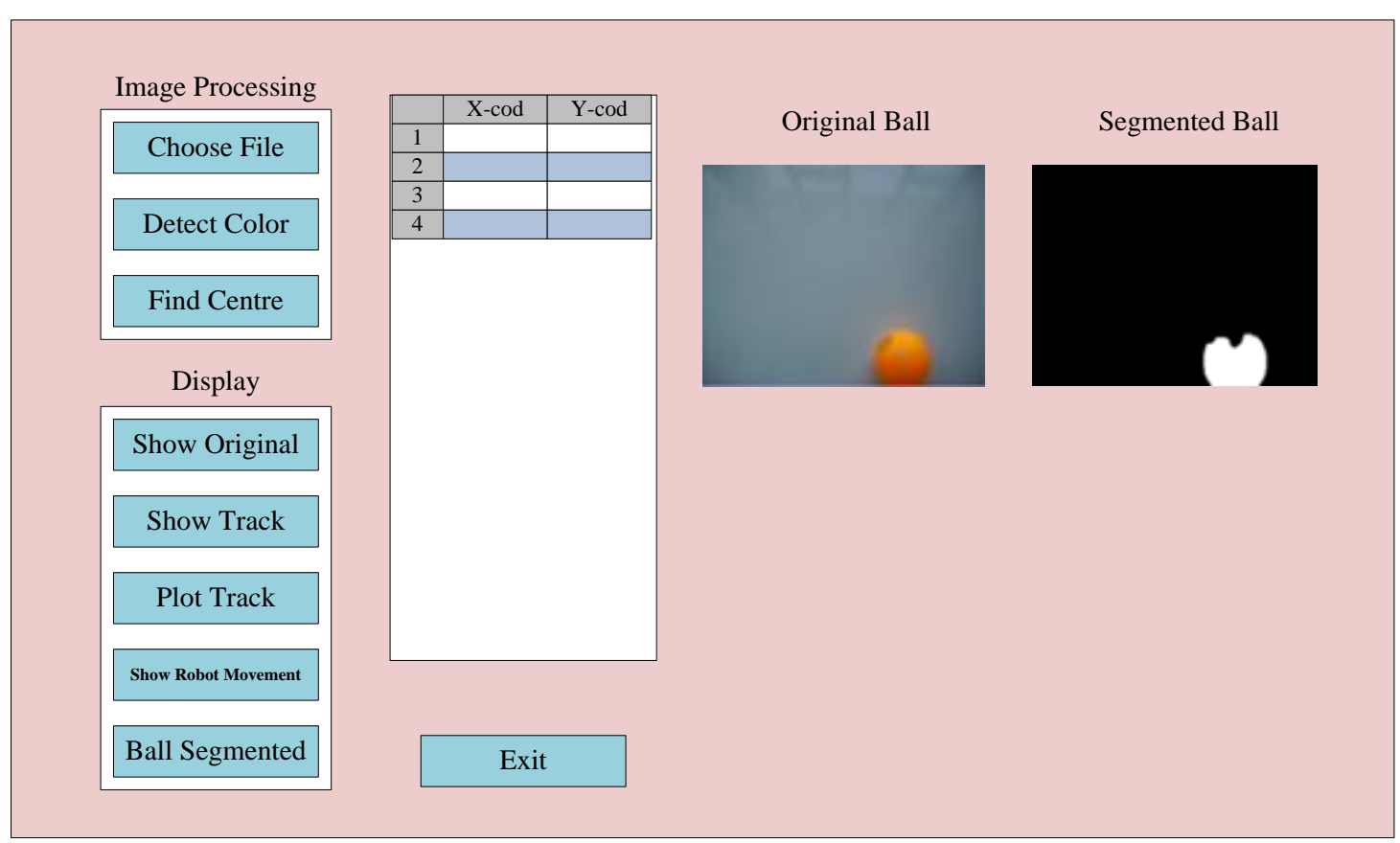

Figure 12. Comparison of Original Image and Segmented Image 


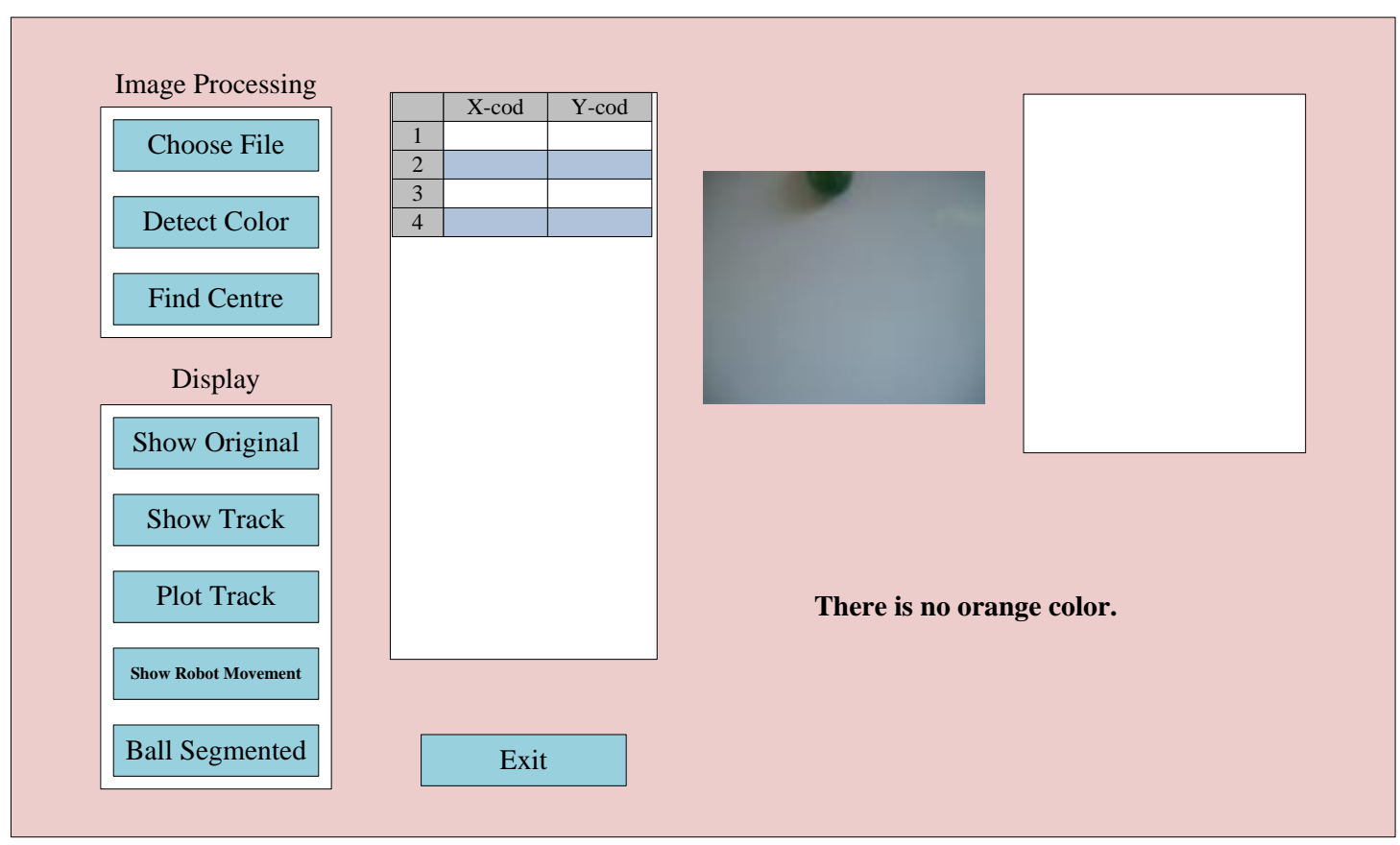

Figure 13. No Detection for Orange Color

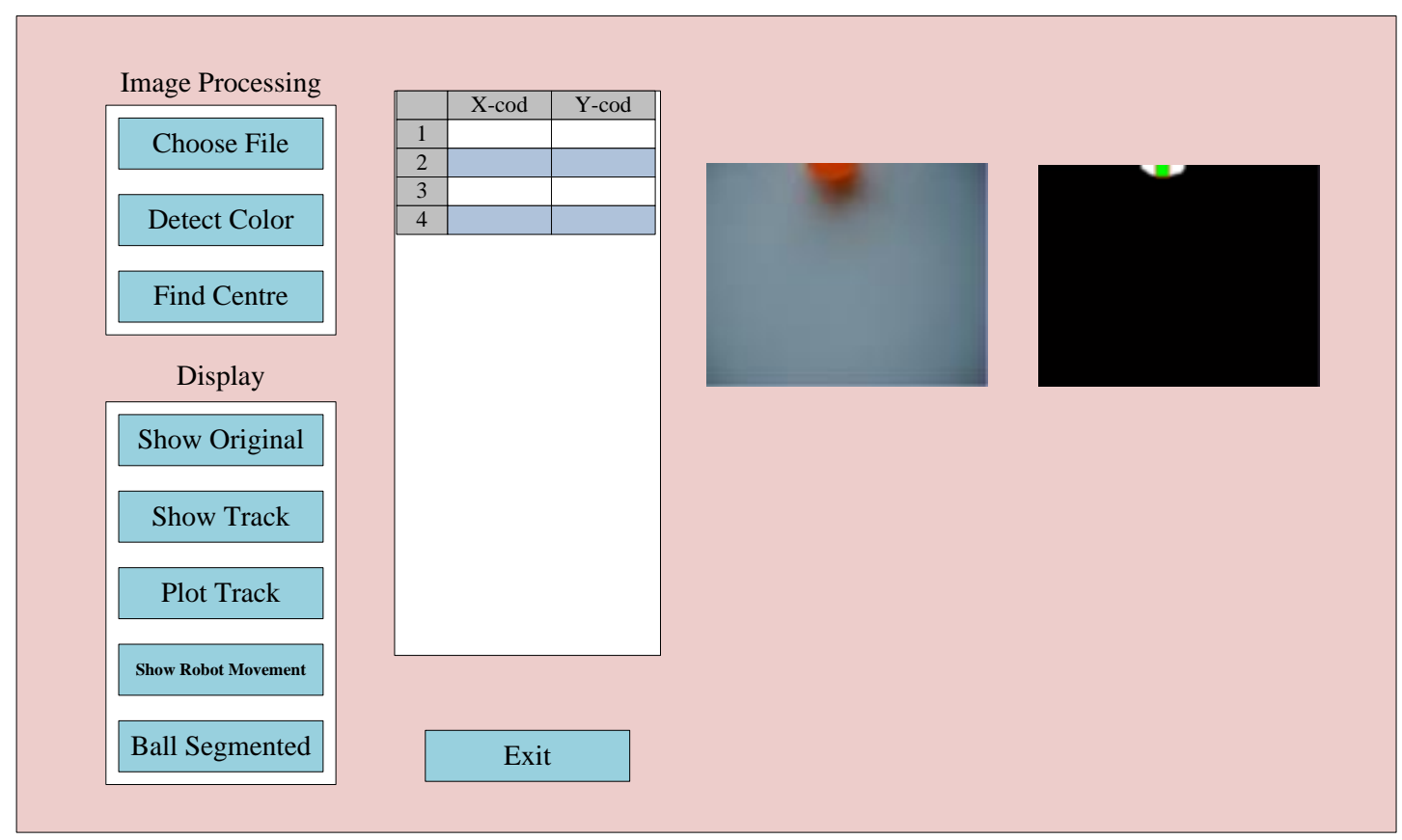

Figure 14. Original Photo and Tracking Path 


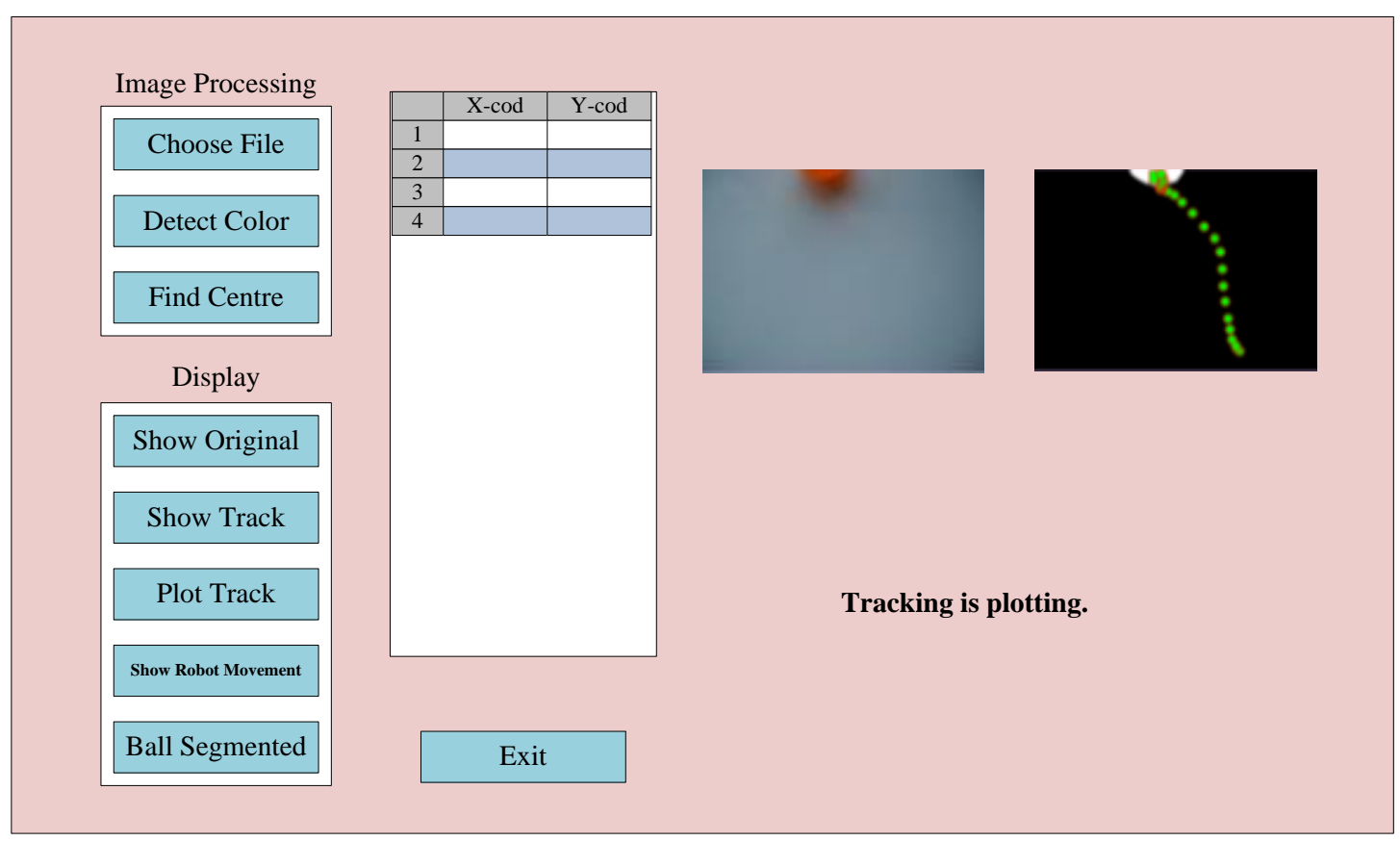

Figure 15. Track Path of the Image

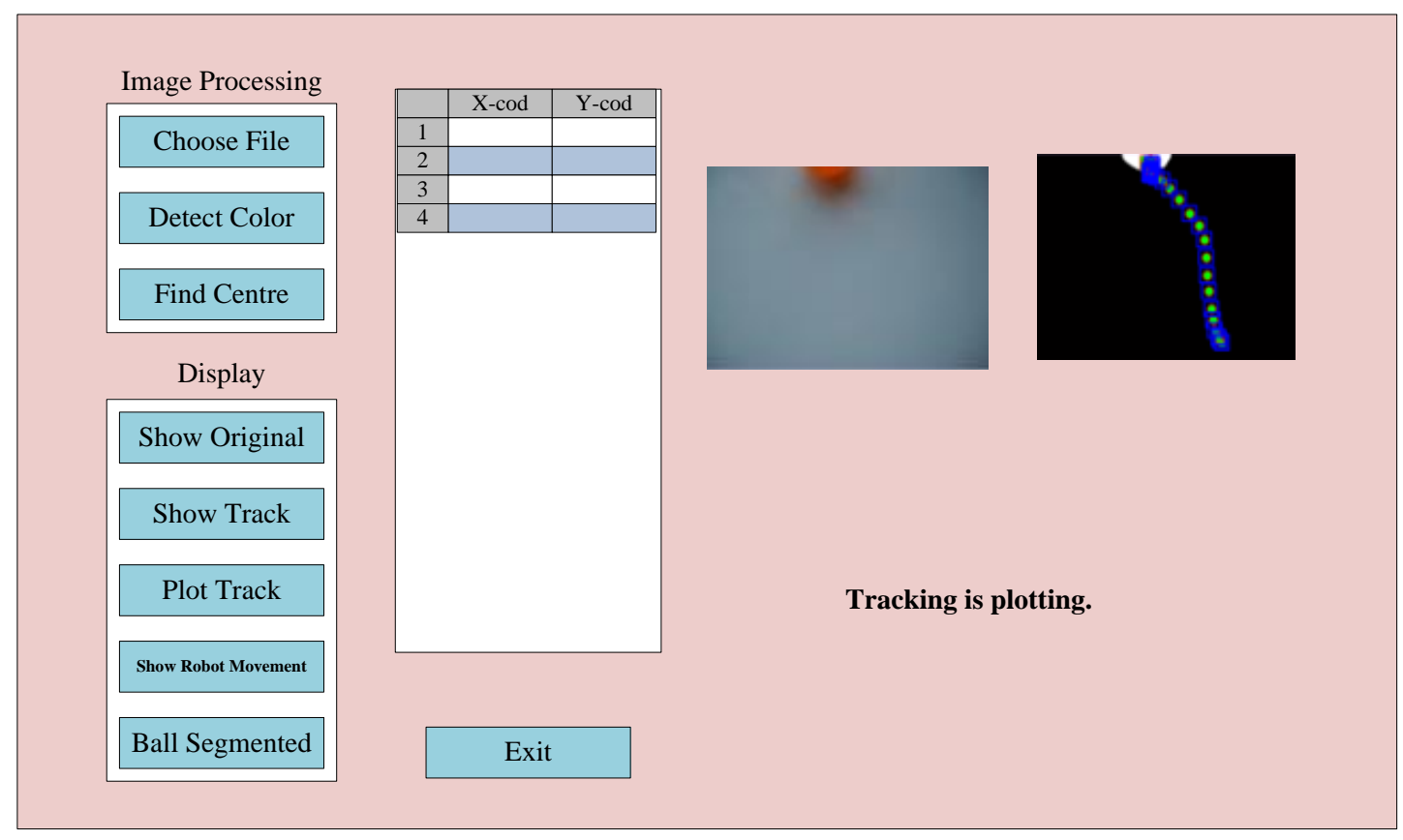

Figure 16. Track Path of the Robot Movement 


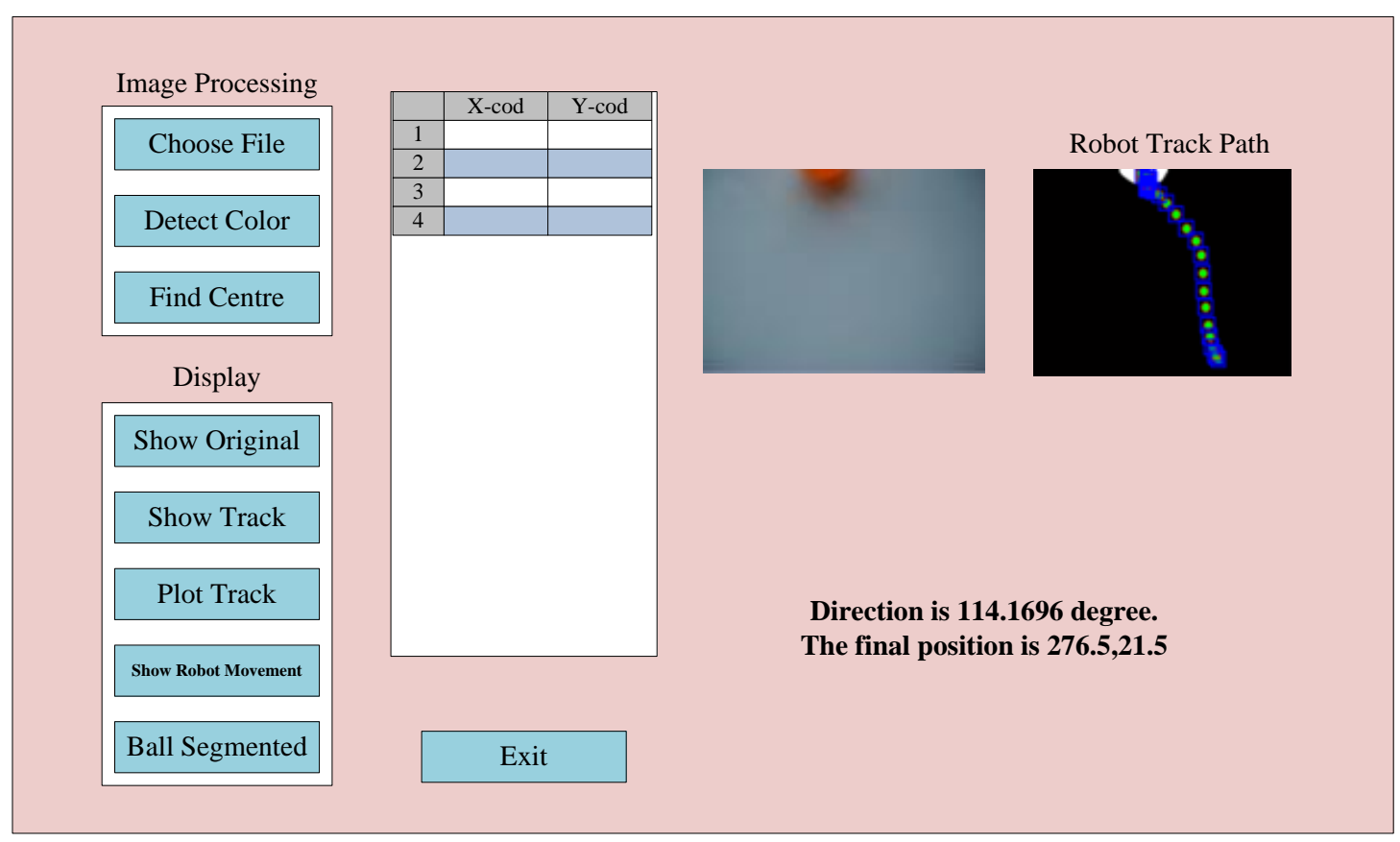

Figure 17. Final Position and Direction

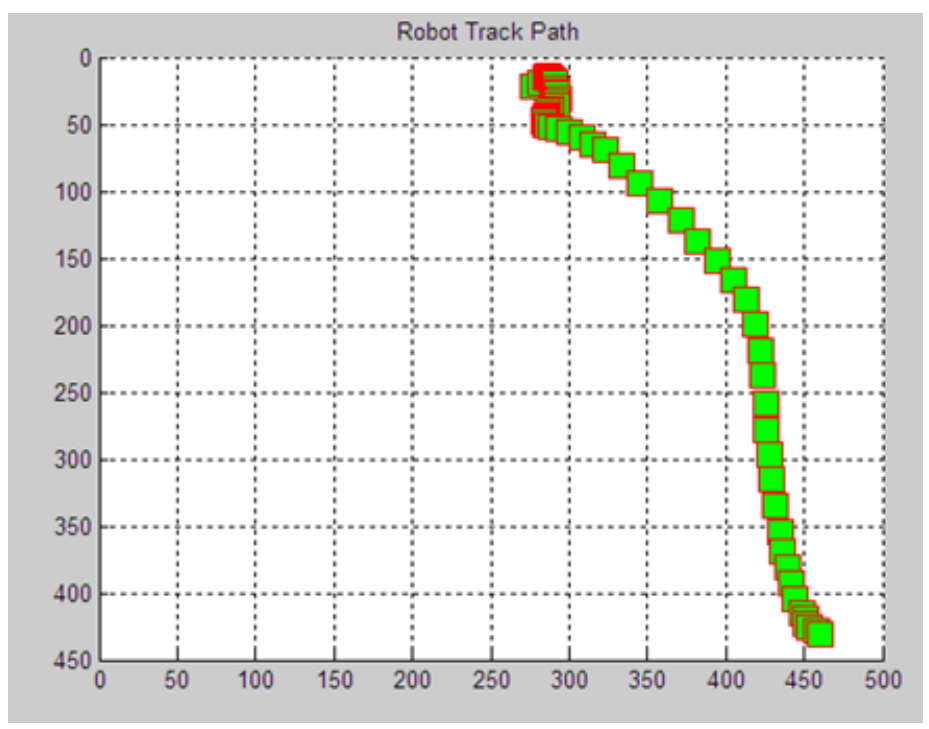

Figure 18. Screenshot of Tracking Path

\section{Summarized Table on Numerical Analysis}

The numerical analysis on the detection of ball is completed by tracked object by using image processing techniques. The numerical evaluation on experimental studies based on the following Table 1 .

Table 1. Summarized Evaluation

\begin{tabular}{cccc}
\hline Condition & Start Point Location & Target Point Location & Accuracy $(\%)$ \\
\hline 1 & 250 & 500 & $75 \%$ \\
2 & 255 & 450 & $95 \%$ \\
3 & 300 & 470 & $85 \%$ \\
\hline
\end{tabular}




\section{Conclusion}

The numerical analysis on tracking algorithm in real time condition confirms that the condition 2 is the highest accuracy value among other conditions. The specific solution for research problem has been proofed for the application in real world situation. The algorithms for image processing are accustomed excerpt a set of structures from the image to characterize an object. Color component analysis is used. For evaluating the components values of each pixel color tracking system is playing in the real world. Using color tracking for a target object controlling has convinced benefits completed additional old-fashioned astronomical tracking schemes. The tracking system is also reasonable and has a minutest of imprint on the further spaceship systems. This system is useful in application such as automatic control system, machine vision system and robotic control system. In automatic control system, it is able to track and control the attitude and position of the object.

\section{Acknowledgments}

The author would like to thank many colleagues from the Department of Electronic Engineering of Yangon Technological University to complete this work.

\section{References}

John, C. R. (1999). The Image Processing Handbook (6th ed.). Materials Science and Engineering Department, North Carolia State University Raleigh, North Carolia, USA.

Miler, D. P., \& Wright, A. (1995). Autonomous Spacecraft Docking Using Multi-Color Targets. Proceedings of the 6th Topical Meeting on Robotics, Monterey, CA, USA.

Slawomir, B. W. (1999). Color Image Edge Detection and Segmentation: A Comparison of the Vector Angle and the Euclidean Distance Color Similarity Measures (Master's thesis, University of Waterloo, Ontario, Canada). Retrived from https://uwspace.uwaterloo.ca/bitstream/handle/10012/937/swesolko1999.pdf?sequence=1\&isAllowed=y

Tim, M. (2004). Computer Vision and Image Processing. Red Globe Press, USA.

Wie, B., \& Reston, V. (1998). Space Vehicle Dynamics and Control (1st ed.). American Institude of Aeronautics and Astronautics, Inc. USA.

William, K. P. (2000). Digital Image Processing (4th ed.). Wiley-Interscience, USA.

\section{Copyrights}

Copyright for this article is retained by the author(s), with first publication rights granted to the journal.

This is an open-access article distributed under the terms and conditions of the Creative Commons Attribution license (http://creativecommons.org/licenses/by/4.0/). 